\title{
Genetic mapping of X-linked loci involved in skewing of $X$ chromosome inactivation in the human
}

\author{
A nna K Naumova ${ }^{1}$, Leonard Olien ${ }^{2}$, Lynne M B ird ${ }^{3}$, M ark Smith ${ }^{1}$, A ndrei E Verner ${ }^{1}$, \\ M ark Leppert ${ }^{4}$, Kenneth M organ ${ }^{2}$ and Carmen Sapienza ${ }^{1,5}$ \\ ${ }^{1}$ Fels I nstitute for Cancer Research and M olecular B iology, Temple U niversity School of M edicine, Philadelphia, \\ USA \\ ${ }^{2} \mathrm{D}$ epartments of $\mathrm{H}$ uman $\mathrm{G}$ enetics and M edicine, McGill U niversity, and M ontreal G eneral H ospital Research \\ Institute, M ontreal, Canada \\ ${ }^{3}$ D ivision of G enetics, Children's H ospital and H ealth Center, San Diego, CA, USA \\ ${ }^{4} \mathrm{E}$ ccles Institute of Human G enetics, U niversity of U tah, Salt L ake City, U tah, USA \\ ${ }^{5} \mathrm{D}$ epartment of Pathology and L aboratory M edicine, Temple U niversity School of M edicine, Philadelphia, USA
}

\begin{abstract}
We have analyzed X-chromosome inactivation patterns in lymphocytes of 264 females from 38 families not known to have any genetic disease. Quantitative measures of $X$-inactivation showed strong sister-sister correlation in the degree of departure from equal numbers of cells having each $X$ chromosome active, suggesting heritability of this phenotype. Strong sister-sister correlation was also observed for the fraction of cells having the same parent's $X$ chromosome active, consistent with the possibility that this trait might be controlled by a cis-acting, X-linked gene. We used a sib-pair approach to determine whether $X$-inactivation phenotype was linked to loci in any region of the $\mathrm{X}$ chromosome. Both quantitative and discrete measures of $\mathrm{X}$-inactivation phenotype showed evidence of linkage to markers in the region of the $X$ inactivation center $(X I C)$. The quantitative measure of $X$-inactivation phenotype used in our study also showed linkage to loci at Xq25-q26. This study provides the first evidence for $X$-linked inheritance of $X$ chromosome inactivation phenotype derived from linkage analysis in phenotypically normal human families.
\end{abstract}

Keywords: skewed X-inactivation; mapping; sib-pair analysis

Correspondence: Carmen Sapienza, Fels Institute for Cancer R esearch and Molecular Biology, Temple U niversity School of Medicine, $3307 \mathrm{~N}$ B road Street, Philadelphia, PA 19140, USA. Tel: (215) 707-7373; Fax: (215) 707-1454; E-mail: CA RMEN@SGI1.FE LS.TEM PLE .EDU

R eceived 9 J une 1998; revised 22 July 1998; accepted 5 A ugust 1998 


\section{Introduction}

O ne of the two $X$ chromosomes in each somatic cell of female mammals is inactivated early in embryonic development. ${ }^{1}$ The choice of which $X$ chromosome to inactivate in any particular cell has a strong stochastic component but both epigenetic and genetic factors have been shown to influence the outcome of this process (see Belmont for review). ${ }^{2}$ In both human ${ }^{3-5}$ and mouse, ${ }^{6,7} \mathrm{X}$ chromosome inactivation is imprinted in early embryonic development such that the paternal $X$ chromosome is inactivated in most cells of extraembryonic lineages. In the mouse, an allelic series at the $X$ ce locus has been demonstrated to affect the probability with which a particular $X$ chromosome is inactivated in somatic cells of the embryo proper. ${ }^{8}$ In contrast, the choice of which $X$ chromosome is inactivated in the human is often said to be 'random'; meaning that on average, the maternal $X$ chromosome is active in one half of the cells of an individual female and the paternal $X$ chromosome is active in the other half. ${ }^{1}$

Individual females who deviate strongly from a 'random' pattern have been described, and familial clustering of females with highly skewed patterns of $X$-inactivation has also been observed (see reviews by B elmont ${ }^{2}$ and Willard ${ }^{9}$ ). Plenge et al ${ }^{10}$ used a candidate gene approach to identify two families in which skewed $\mathrm{X}$-inactivation phenotype co-segregated with a specific allele at the XIST locus, which resides within the cytogenetic region defined as the $X$ inactivation center, ${ }^{11}$ or XIC. However, skewed X-inactivation phenotype cannot be demonstrated to map to the XIC in all families or individuals that show the trait, ${ }^{12-16}$ despite evidence for the involvement of an $\mathrm{X}$-linked gene in some of these instances. ${ }^{16,17}$ In these cases, one cannot distinguish between models that invoke genetic control of a step in the $X$ chromosome inactivation pathway and models that invoke selection for cells that have a particular $\mathrm{X}$ chromosome in the active or inactive state. ${ }^{18,19}$

Virtually all reports demonstrating 'non-random' $X$-inactivation presuppose that the cases described are likely to be exceptional and there are few indications that normal variation in $X$ chromosome inactivation is heritable. However, failure to observe inheritance of this trait cannot be taken as strong evidence that it is not heritable because many factors might mask our ability to make this observation. Selection of the genetic model, unknown penetrance of the trait, the potential presence of phenocopies within families and the possibility of genetic heterogeneity ${ }^{11,17,20-22}$ are all likely to be complicating factors. We have attempted to circumvent some of these difficulties by using a sib-pair approach to examine the heritability of $X$-inactivation phenotype in 264 females from 38 families and to search for linkage to loci on the $\mathrm{X}$ chromosome. $\mathrm{O}$ ur analyses, which are largely independent of mode of inheritance, indicate that $X$ chromosome inactivation phenotype is heritable and is linked to loci within or near the XIC at $\mathrm{Xq13}-\mathrm{q} 21$ and to loci at X q25-q26.

\section{Materials and Methods}

\section{Quantitation of X-inactivation Skewing}

The $X$-inactivation phenotype of individual females was scored using the PCR -based assay ${ }^{23}$ for differential methylation of alleles at the androgen receptor locus (AR), as used in our previous studies. ${ }^{17,24}$ D etermination of $X$-inactivation by the FRAXA methylation PCR assay ${ }^{25}$ was done, in addition, on one family, K 1362, using primers ' $\mathrm{C}$ ' - 5 ' GCTCAGCTCCGTTTCGGTTTCACTTCCGGT 3 ' and ' $f$ ' 5' AGCCCCGCACTTCCACCACCAGCTCCTCCA 3'. $Q$ uantitation of the results of the FRAXA methylation assay was performed by laser-scanning densitometry or phosphorimaging analysis, as described for the $A R$ methylation assay. ${ }^{17,24}$

We derived two measures of $\mathrm{X}$-chromosome inactivation skewing:

1 The active proportion of the maternally inherited chromosome, denoted $\mathrm{PA}_{\text {mat }}$

$$
\mathrm{PA}_{\text {mat }}=[\mathrm{m} /(\mathrm{m}+\mathrm{s})]^{\mathrm{Hha}+}
$$

where $\mathrm{m}$ is the densitometer quantitation or phosphorimage count corresponding to the paternal allele, and $s$ is the value for the maternal allele, of the androgen receptor PCR product after the template has been digested with $\mathrm{H}$ hal. For certain individuals whose $A R$ alleles differed in length by only one trinucleotide repeat, the $\mathrm{PA}_{\text {mat }}$ score was adjusted by $[\mathrm{m} /(\mathrm{m}+\mathrm{s})]^{\mathrm{H} \text { ha- }}$, as in Naumova et al. ${ }^{24}$ The $P A_{\text {mat }}$ score measures both the magnitude and direction of skewing. $A$ $\mathrm{PA}_{\text {mat }}$ score of 0.5 indicates no skewing, a PA mat score of zero indicates that all cells have an active paternal chromosome, whilst a PA mat score of 1 indicates that all cells have an active maternal chromosome.

2 The degree of skewing score, denoted DS, is a measure of only the magnitude of skewing. ${ }^{24}$ Zero indicates no skewing and 0.5 indicates complete skewing; that is, the active chromosome in every cell is of the same parental origin.

$D S=\left|\left(P A_{m a t}-0.5\right)\right|$

\section{Correlation of Trait Values}

We tested for sister-sister correlation and for motherdaughter correlation in both the DS score and the PA $\mathrm{A}_{\text {mat }}$ score. The sister-sister correlation was estimated from sibships with at least two sisters using analysis of variance. ${ }^{26}$ Significance was assessed using the permutation method ${ }^{27}$ 
because sibship sizes were small, and the trait is not normally distributed (see Figure 1 in Naumova et $\mathrm{al}^{17}$ ). Permutation replicates were generated by assigning individual females to sibships randomly, without replacement, from the pool of all

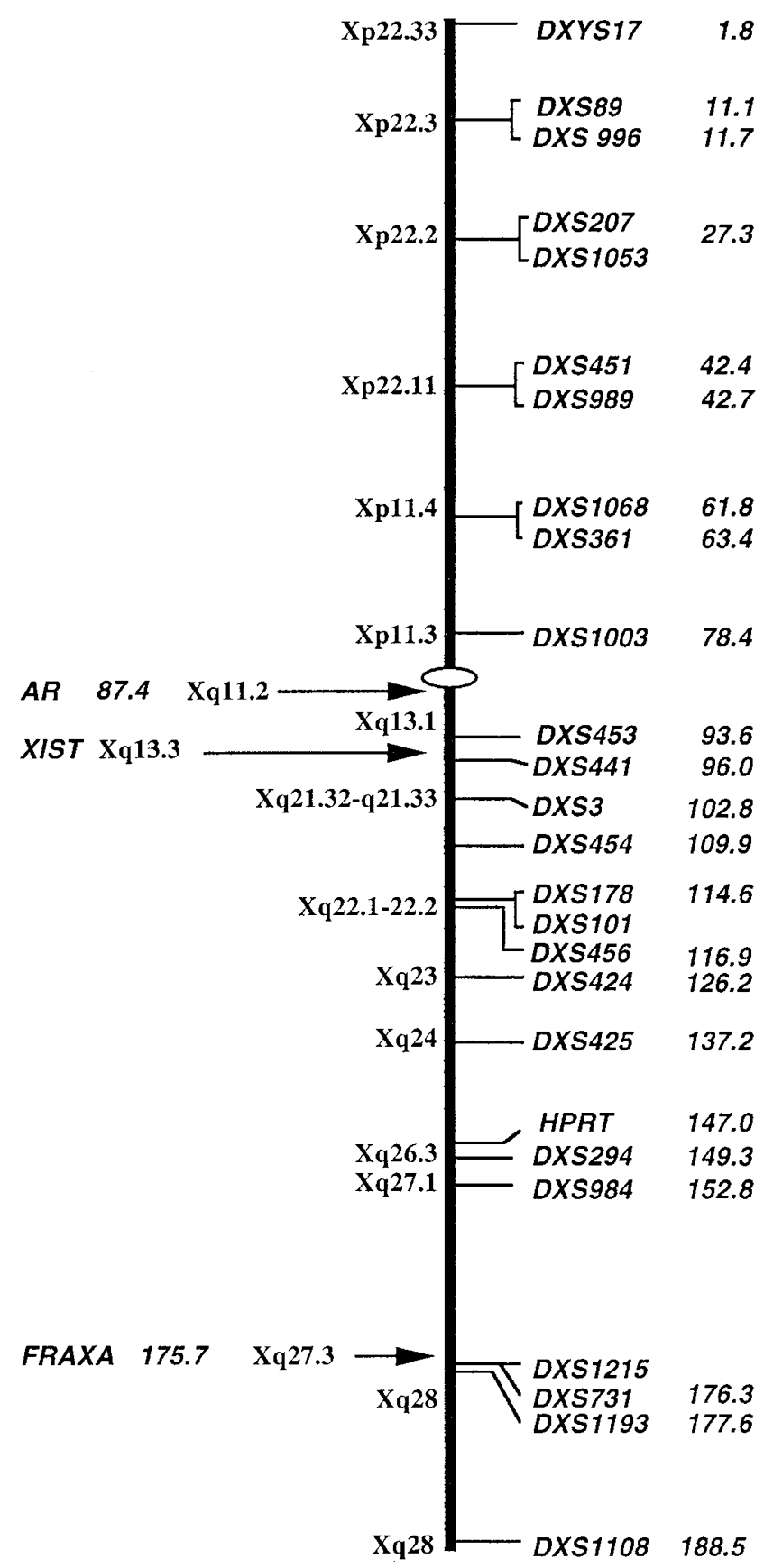

Figure 1 G enetic map of the human $X$ chromosome. $D$ istance between markers and marker order, with the exception of DXS101, are taken from the linear version of the female $X$ chromosome map $^{31}$ (see GenL ink public database [http:/ /www.genlink.wustl.edu]). D istances are displayed in Kosambi centiM organs. DXS101 has been placed distal to DXS178 according to Vetrie et al. ${ }^{42}$ females in the third generation. The distribution of the test statistic under the null hypothesis was found by calculating the test statistic for each of 2000 replicates. The empirical significance level (or achieved significance level, ${ }^{27}$ ) was defined as the proportion of permutation replicates with sister-sister correlation greater than the value calculated from the actual sample.

A nalysis of the mother-daughter correlation also utilized the permutation method. In this case, a permutation replicate was generated by randomly assigning mothers to families, sampling without replacement, from the pool of all mothers. In this way, the distribution of the mother-daughter correlation is conditioned on the correlation structure of the daughters. We used two estimators of the mother-daughter correlation: the pairwise estimator and the sib-mean estimator. ${ }^{26,28}$ The pairwise estimator matched each daughter's score with the mother's score, whilst the sib-mean estimator matches the mean of a sibship with the score of the mother. The pairwise estimator performs well for low sibling correlation but gives greater weight to larger families. ${ }^{26}$ The sib-mean estimator performs well for higher sibling correlation but has larger asymptotic standard error than the pairwise estimator. 28

\section{Linkage Analysis Using Sib-pairs}

We used the Haseman-EIston sib-pair approach ${ }^{29}$ to test for linkage of the $P A_{\text {mat }}$ score to 15 markers on the $X$-chromosome. D efine $Y_{j}=\left(y_{i 1}-y_{j 2}\right)^{2}$, where $y_{i j}$ is the $P A_{\text {mat }}$ score of the ith member of the jth pair of sibs. In the standard approach, $Y_{j}$ is regressed on $p_{j}$, the proportion of alleles at $a$ given locus shared identical-by-descent (IBD) by the jth pair of sibs. In the present study, for each marker, individuals were included for whom the genotype was known or could be assigned with $95 \%$ probability (see below), therefore $p_{\text {. }}$ was estimated by counting. Furthermore, $p_{\text {w }}$ will equal either $1 / 2$ or 1 for a pair of female sibs since they will have inherited either the same or different maternal alleles but will always inherit the same paternal allele. Therefore $\mathrm{H}$ aseman-E Iston analysis is equivalent, in this case, to performing a test on $b=m_{2}-m_{1}$, where $m_{k}$ is the mean of the $Y_{j}$ 's for those sib-pairs that share $\mathrm{k}$ alleles IBD. Under the hypothesis of no linkage, the two groups will have identical means $(b=0)$. H owever, if the trait is linked to a particular marker, then $b$ will be negative; that is, the alternative hypothesis is $b<0$.

Significance of linkage to a given $X$ chromosome locus was assessed using the parametric bootstrap method ${ }^{27}$ on the maternally inherited allele for each daughter. U nder the null hypothesis of no linkage, each daughter receives the grandpaternal allele from her mother with probability $1 / 2$. We generated bootstrap replicates by randomly assigning, to each daughter, the grandparental origin of the maternally inherited allele. Two thousand replicates were generated and b was calculated for each replicate, which provided the distribution of $b$ under the null hypothesis conditional on the sample structure. The empirical significance level was defined as the proportion of bootstrap replicates with $b$ less than that of the actual sample. Because we bootstrapped marker alleles, the observed distribution of sibship sizes and sister-sister correlation of the trait were maintained for every replicate. Therefore, it was not necessary to adjust the statistic for nonindependence of the sib-pairs since all replicates were generated under the same structure as the observed sample (see $\mathrm{H}$ auser et $\mathrm{al}^{30}$ ). 
Fifteen loci were chosen along the entire $\mathrm{X}$-chromosome so that, where possible, adjacent markers were separated by not more than $15 \mathrm{~cm}$. Where data were not available for a particular marker in a given individual, nearby markers were typed or retrieved from the GenLink database ${ }^{31}$ (http:/ /www.genlink.wustl.edu). The genotype at the marker of interest was then inferred according to the following criteria. The probability that the missing allele was of grandpaternal origin was calculated using flanker markers and joint recombination probabilities. ${ }^{32}$ The missing allele was assigned to be of grandpaternal origin if the probability was greater than 0.95 , whereas the allele was assigned to be of grandmaternal origin if the probability was less than 0.05 . The data used in the linkage analysis comprised 15 markers and 76 individuals. Of the 1140 observations, 707 were typed by our lab or retrieved from the database, 328 were inferred as above, and 105 observations were uninformative for our analysis.

\section{Permutation Test for Familial Aggregation of Skewing as a Discrete Trait}

To test for familial aggregation of skewing, a contingency table of skewing status (the quantitative phenotype was transformed to the discrete trait 'skewed' or 'nonskewed' by designating individuals with at least $80 \%$ of their cells having the same X chromosome active as 'skewed') versus family was constructed using third-generation females. All families in which the skewing score was available for at least two sisters were included. Significance was estimated using the permutation method. ${ }^{27}$ In this case, the null hypothesis is that there is no familial aggregation of skewing, that is skewing is independent of family. The test statistic is the probability of the contingency table, as calculated from the hypergeometric distribution. ${ }^{33}$ Two thousand permutation replicates were generated at each threshold value. Each replicate was generated by randomly assigning individuals to families, while maintaining the distribution of sibship sizes. The empirical significance level is defined as the proportion of random permutation tables with probabilities less than or equal to the probability of the observed table. A $n$ empirical significance level of 0.05 was used to reject the null hypothesis of no familial aggregation of skewing.

\section{Subjects Used in the Sibling Concordance Study}

Five of the 38 families used in our initial screening were selected for the sibling concordance analysis on the basis of having two or more siblings who exhibited at least $80 \%$ of cells with the active $X$ chromosome inherited from the same parent. Four of these five families came from the extended CEPH families collection and one family was collected by our laboratory. DNA from lymphocytes was used for $X$-inactivation assays, whereas DNA from lymphocytes and/ or lymphoblastoid cell lines was used for determining the genotypes of the individuals at the $\mathrm{X}$-chromosome loci tested (see below).

In our discrete trait mapping study, we considered the genotypes of only skewed females. Our rationale for this decision is that there are a number of ways, both genetic and non-genetic, in which a daughter who inherited an X-linked gene for skewing might fail to exhibit the skewing phenotype (inheritance of alleles at other loci that might counteract skewing, individual variation in the allocation of hematopoietic stem cells with one or the other $\mathrm{X}$ chromosome active, environmentally mediated counter selection, truncation selection imposed by transformation of the quantitative phenotype to a qualitative phenotype, etc.). H owever, given the results of our analyses of familial correlation and aggregation of skewing (R esults and unpublished data), and our previous analysis of the frequency of skewing in unrelated females, ${ }^{17}$ we regarded it as unlikely that skewing in multiple females within the same family as a result of non-genetic factors would be a serious concern.

\section{Genotype Determination}

$G$ enotypes of all subjects at loci on the $X$ chromosome were determined by PCR at markers spanning the length of the $X$ chromosome. In all cases, we attempted to keep the distance between informative markers to less than $15 \mathrm{cM}$. Oligonucleotide PCR primers for the loci shown in Figure 1 were purchased from Research Genetics, Inc. (H untsville, $A L$, U SA ), with the exception that primers for the A R locus were kindly provided by Dr K im D eR iel, and primers for D X S294, DXS984, and FRAXA were purchased from Genosys Biotechnologies (The Woodlands, TX, USA). In cases where individuals were not informative for the markers listed above, we used data for closely linked markers from the public database, where it was available. Thus, the data for markers DXS89, DX S342, DXS451，DXS1048，DXS319，DXS361, DXS453, DX S456, DXS425, HPRT, and some of the data for DXS989 and DXS1108 for families K 1332, K 1341, K 1347, $\mathrm{K} 1375$ and K 1362 were taken from the GenLink database [http://www.genlink.wustl.edu, data release January $1996^{31}$ ]. The genotypes determined in our laboratory were compared, where possible, with those from the public database and no discrepancies were found.

\section{Results}

\section{Skewed X-inactivation as a Quantitative Trait}

We have used allele-specific methylation of CpG sites within the androgen receptor (AR) locus as a quantitative measure of $X$ chromosome inactivation ${ }^{23}$ ( $M$ aterials and $M$ ethods). Two quantitative measures of $X$-inactivation were derived from these measurements. The 'degree of skewing' score (DS), measures only the absolute magnitude of 'skewing' and the PA mat score takes into account both the magnitude and the direction (proportion of cells with the maternal $X$ chromosome active) of skewing. The D S score is expected to be responsive to any genetic factor that affects skewing per se, whilst the PA $A_{\text {mat }}$ score is expected to be responsive to any $X$-linked gene that acts in cis, or any gene that exerts a parent-of-origin effect on $X$-inactivation.

A II tests for sister-sister and mother-daughter correlations in DS score and $\mathrm{PA}_{\text {mat }}$ score were performed 
Table 1 Sister-sister correlation in DS and PA mat $_{\text {scores }}$

\begin{tabular}{llc}
\hline Statistic & PA $_{\text {mat }}$ & DS \\
\hline N umber of sisters & 98 & 100 \\
N umber of sibships & 26 & 27 \\
Correlation & 0.3791 & 0.2462 \\
P-value & 0.0000 & 0.0025 \\
\hline
\end{tabular}

${ }^{\mathrm{P}} \mathrm{P}$-value determined by permutation test using 2000 replicates.

independently. Each test used 2000 random permutation replicates.

\section{Sister-sister Correlation in X-inactivation Phenotype}

We tested the hypothesis that there was no sister-sister correlation in the DS score or the PA $A_{\text {mat }}$ in 38 families (Table 1). For the DS score, only five of the 2000 permutation replicates (empirical significance level $=0.0025$ ) had a sister-sister correlation greater than that of the observed families. For the PA ${ }_{\text {mat }}$ score, none of the permutation replicates had a sister-sister correlation greater than that found between sisters in the observed families. Thus, we reject the null hypothesis of no sister-sister correlation for both the D S score and the $\mathrm{PA}_{\text {mat }}$ score. The correlation in DS score indicates that the $\mathrm{X}$-inactivation phenotype measured in siblings is not random, but that sisters are more similar to each other than are unrelated females. Because the PA mat score also takes into account the parental origin of skewing, i.e. whether sisters have the same proportion of cells with the maternal $X$ chromosome active, these data also suggest that if a genetic factor is influencing $X$-inactivation phenotype, that factor may lie on the $X$ chromosome and act in cis.

\section{Lack of Mother-daughter Correlation in $X$-inactivation Phenotype}

We also tested for mother-daughter correlation in the $D S$ and $P A_{\text {mat }}$ (Table 2). N one of the $P$ values were less than 0.05 , therefore we do not reject the null hypothesis of no mother-daughter correlation in $D S$ or $P A_{\text {mat }}$

Table 2 M other-daughter correlation in D S and PA $A_{\text {mat }}$ scores

\begin{tabular}{llr}
\hline Statistic & $\mathrm{PA}_{\text {mat }}$ & $\mathrm{DS}$ \\
\hline N umber of daughters & 75 & 82 \\
N umber of mothers & 21 & 22 \\
Pairwise estimator: & & \\
$\quad$ Correlation & 0.0720 & -0.0599 \\
$\quad$ P-value & 0.3800 & 0.6305 \\
Sib-mean estimator: & & \\
$\quad$ Correlation & 0.0364 & -0.0261 \\
P-value & 0.4540 & 0.5490 \\
\hline
\end{tabular}

${ }^{\mathrm{P}} \mathrm{P}$-value determined by permutation test using 2000 replicates. score. These data indicate that, overall, mothers and daughters within the same family are no more similar in their $\mathrm{X}$-inactivation phenotype than random permutations of mothers among the families. The interpretation of this observation with regard to genetic control of $X$-inactivation phenotype is, in large part, dependent on the genetic model by which $X$-inactivation phenotype is assumed to be inherited and expressed (see $D$ iscussion), but these data indicate that $X$-inactivation phenotype is not the result of a simple, $X$-linked dominant or autosomal dominant mode of inheritance.

\section{Mapping X-linked Genes that Influence $X$-inactivation by Haseman-Elston Sib-pair Analysis}

Because of our analysis of the PA mat score (Table 1 ) indicated that an $\mathrm{X}$-linked gene might be involved in controlling this trait in cis, we modified the HasemanElston sib-pair approach of linkage analysis for this particular trait ${ }^{29}$ ( $M$ aterials and Methods). We determined the genotypes of each female at the $X$ chromosome loci shown in Figure $1 .{ }^{31}$ The number of sib-pairs, b statistic and empirical significance level are shown in Table 3. Four markers are linked to $X$-inactivation phenotype at a significance level of 0.05 or less. Two, DXS453 and DXS441 are closely linked to each other (approximately $3 \mathrm{cM}$ apart, ${ }^{31}$ see Figure 1 ) and are located at $X q 13$ in the region of the XIC. Two additional markers that are linked to $\mathrm{X}$-inactivation

Table 3 L inkage analysis of $X$-inactivation skewing

\begin{tabular}{llll}
\hline M arker & $\mathrm{N}$ & $\mathrm{b}$ & $\mathrm{P}$-value \\
\hline DXS996 & 94 & 0.032 & 0.894 \\
DXS207 & 104 & 0.003 & 0.558 \\
DXS989 & 86 & 0.0076 & 0.627 \\
DXS1068 & 100 & -0.016 & 0.237 \\
DXS1003 & 100 & -0.0329 & 0.103 \\
AR & 120 & -0.0299 & 0.0855 \\
DXS453 & 116 & -0.049 & 0.0175 \\
DXS441 & 112 & -0.0519 & 0.0195 \\
DXS3 & 112 & -0.0214 & 0.1715 \\
DXS101 & 99 & -0.0066 & 0.3585 \\
DXS424 & 112 & -0.0068 & 0.359 \\
DXS425 & 101 & -0.0466 & 0.035 \\
DXS294 & 106 & -0.06 & 0.0075 \\
DXS731 & 111 & -0.0387 & 0.0505 \\
DXS1108 & 83 & 0.0343 & 0.881 \\
\hline Nis thenumber
\end{tabular}

$\mathrm{N}$ is the number of sib-pairs; $\mathrm{b}=\mathrm{m}_{2}-\mathrm{m}_{1}$, where $\mathrm{m}_{k}$ is the mean of the squared difference in trait values of pairs of sibs that share $\mathrm{k}$ alleles IBD at a given locus (see Materials and $M$ ethods); $P$-value is the empirical significance level using the parametric bootstrap and 2000 replicates generated under the null hypothesis. 
phenotype, DXS425 and DX S294, are also linked to each other ( $12 \mathrm{CM}$ apart, ${ }^{3}$ see Figure 1 ) and are located at $X q 25-q 26$. If we adjust the suggested guidelines for reporting the significance of linkage results proposed by $L$ ander and Kruglyak ${ }^{35}$ for the fact that we tested only loci on the $X$ chromosome (the $X$ chromosome contributes approximately $200 \mathrm{cM}$ of the some $3300 \mathrm{cM}$ that make up the human genome), both regions are 'suggestive' of linkage.

\section{Skewed X-inactivation as a Discrete Trait}

\section{Familial Aggregation of $80 \%$ Skewing}

In most studies seeking a genetic basis for variation of $X$ chromosome inactivation in the human, the phenotype has been transformed from an inherently quantitative trait to a discrete trait by specifying a threshold value. Individuals having at least $80 \%$ of their cells with the same $X$ chromosome active have been described as having non-random or skewed $\mathrm{X}$-inactivation in a number of studies. ${ }^{10,17,22,24,34}$ A lthough there is an important historical precedent for choosing the $80 \%$ threshold to designate an individual as having nonrandom $\mathrm{X}$-inactivation ${ }^{7}$ and see discussion in $\mathrm{Nau}$ mova et $\mathrm{al}^{17}$ ) it has not, to our knowledge, been demonstrated that individuals who are skewed to this level constitute a distinct phenotypic group. As one measure of this possibility, we tested whether females who were skewed to this degree or greater were distributed randomly among the 38 families or were clustered within families. 0 nly 25 of the 2000 permutation replicates had a lower probability than the probability for the contingency table of observed families (empirical significance level $=0.0125$ ). These results provide evidence for familial aggregation of skewed $\mathrm{X}$-inactivation, as a discrete trait, when a threshold value of at least $80 \%$ of cells with the same $X$ chromosome active is used.

\section{Mapping of a Locus Involved in a Discrete Phenotype of Skewed X Chromosome Inactivation}

We selected families containing two or more sisters skewed in favor of the same $X$ chromosome active for use in an 'affected' sibling mapping study. Of the 38 three-generation families examined, only five contained two or more sisters who were concordantly skewed to the $80 \%$ level or greater (Figure 2). The skewed sisters in these five families were used to map an $\mathrm{X}$-linked locus that may be responsible for the skewing phenotype. A t each locus for which the sisters were informative, they were scored as concordant if they all inherited the same allele from their mother and were scored as discordant if any of them inherited a different allele. In our mapping strategy, X chromosome regions at which skewed sisters in all five families are concordant may contain genes involved in the skewed $X$-inactivation phenotype, whilst any locus that is discordant in one or more families is excluded. B ecause there may be females who are skewed for nongenetic reasons or because the trait may be genetically heterogeneous, this mapping strategy is likely to be conservative, in that loci are increasingly likely to be excluded as the number of skewed individuals considered becomes larger.

The regions of the $X$ chromosome for which skewed females in each family may be concordant, assuming no recombination between consecutive concordant loci, is shown in Figure 3. Only a small region of the $X$ chromosome is not excluded by one or more of the families. This region is limited on the proximal side by DXS441, within the XIC at Xq13, and on the distal side by $D X S 3$, at X q21. This interval is denoted as 'region of concordance' in Figure 3.

\section{Discussion}

We have examined $\mathrm{X}$-chromosome inactivation phenotype for evidence of heritability in 38 'normal' families. We found a significant correlation between sisters in the degree to which they deviated from having equal proportions of lymphocytes with each $\mathrm{X}$ chromosome active, consistent with the possibility that this quantitative trait has a heritable component. Further evidence in favor of a heritable component of $X$-inactivation phenotype was obtained by comparing sisters for the fraction of their cells having an active maternal $X$ chromosome. A significant sister-sister correlation was found, consistent not only with the heritability of this trait, but also with the possibility that this phenotype is controlled by a cis-acting, $\mathrm{X}$-linked gene.

A Ithough we found evidence for sister-sister correlation in the quantitative trait (Table 1), we found no evidence of mother-daughter correlation in either the DS score or the PA $A_{\text {mat }}$ score (Table2). These results suggest that $X$-inactivation might be inherited in an unusual fashion. For this reason, linkage analysis was performed using a non-parametric method. H asemanE Iston sib-pair analysis provided evidence for linkage 
of the $X$-inactivation trait to loci in two regions of the $X$ chromosome, Xq13 and X q25-q26 (Table 3).

Because there is considerable debate $e^{35-37}$ over the appropriate nominal $\mathrm{P}$-value required to report linkage, it is worth considering the possibility that one or both of the regions showing linkage to $X$-inactivation phenotype are false positive results due only to chance. In this regard, we may offer the following three counterpoints:

1 The sib-pair approach is robust. The method does not specify either a genetic model or a model for the distribution of the quantitative trait that is conditional on genotype, and the bootstrap method does not require assumptions on the distribution of the test statistic;
2 loci in the vicinity of both regions have been identified previously in conjunction with X-chromosome inactivation; ${ }^{10,11,13-16,22,38,39}$

3 we have not conducted a dense scan of the $X$-chromosome, nor have we tested for linkage at each point along the chromosome.

A $n$ additional consideration in interpreting these results is that, strictly speaking, we cannot distinguish whether any linkage identified reflects the activity of genes that might be involved directly in the process of $X$ chromosome inactivation or whether some alleles at these loci might confer a proliferative advantage or disadvantage on the cells in which they are found (see reviews $\left.^{2,9,19}\right)$. However, we note that one of the chromosome regions we have identified as showing
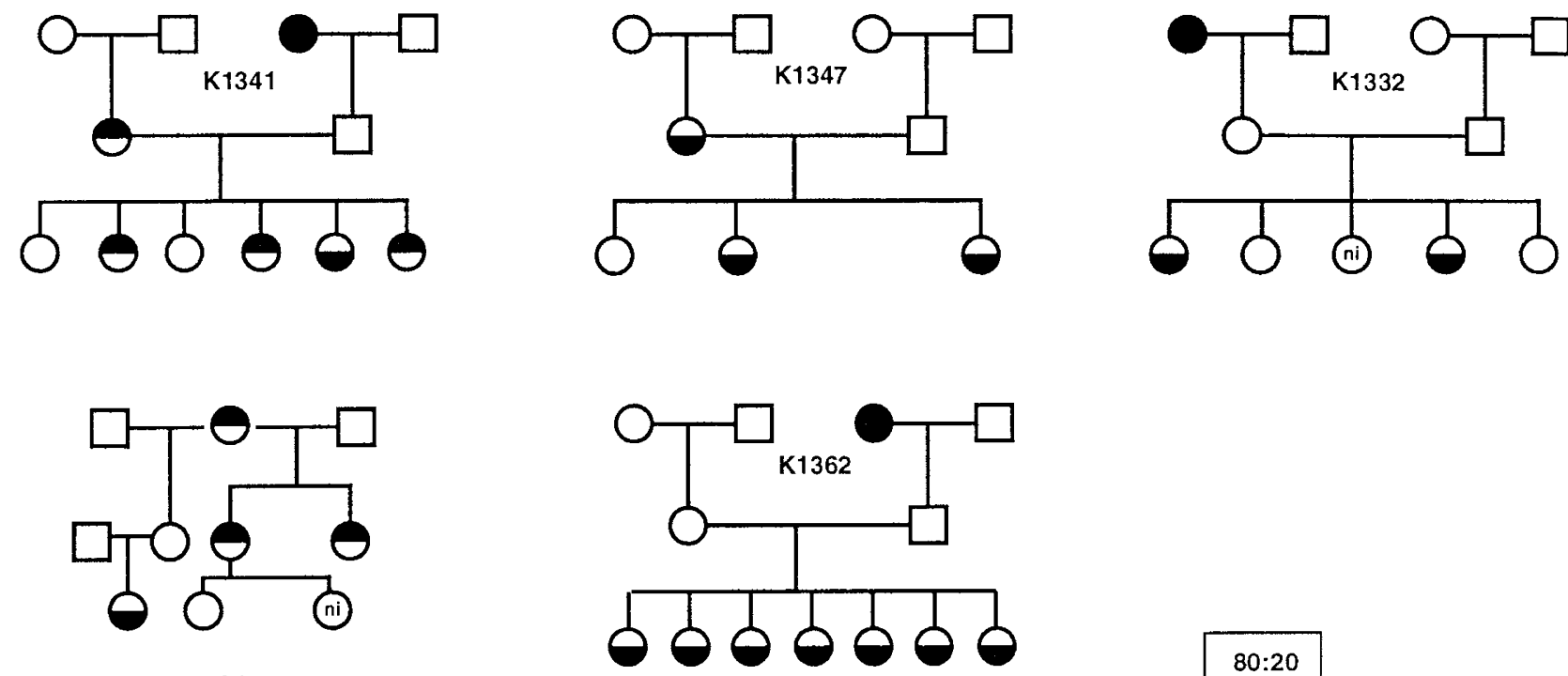

$80: 20$

A1

skewed female, parental origin of the active $X$ unknown

skewing in favor of matemal $X$

skewing in favor of paternal $X$

Figure 2 Families selected for linkage analysis. O nly the females of each family are shown in the bottom generation. O pen circles - 'not skewed' females (see text), half-filled circles - 'skewed' females, ni - not informative for the AR locus or DNA not available. 
evidence for linkage to the quantitative trait (X q13) contains the cytogenetically defined $X I C,{ }^{38,39}$ as well as the XIST gene. ${ }^{11}$.

We also transformed the $X$-inactivation phenotype from a quantitative trait to a discrete trait by designating those females with the same $X$ chromosome active in at least $80 \%$ or their cells as skewed and selecting families for analysis on the basis of having two or more sisters skewed in favor of the same $X$ chromosome active. Five of the 38 families fit these criteria and we used these families in a sibling concordance, exclusion mapping study. The only region of the $X$ chromosome

a) K1332(2)
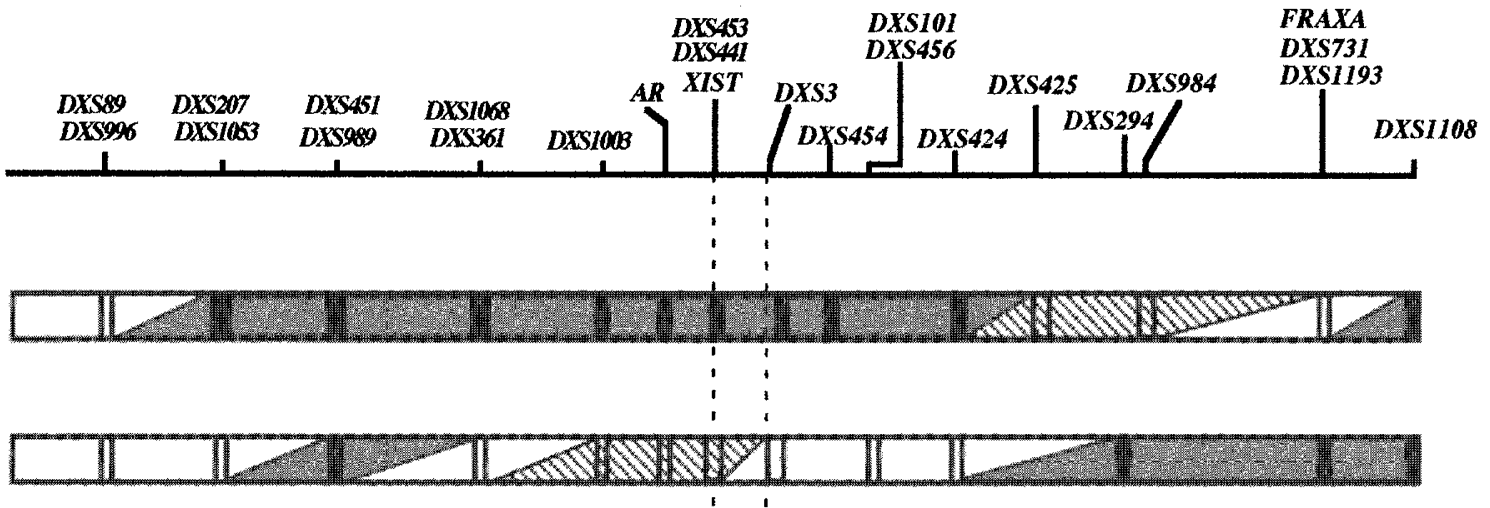

i

K1341(3)

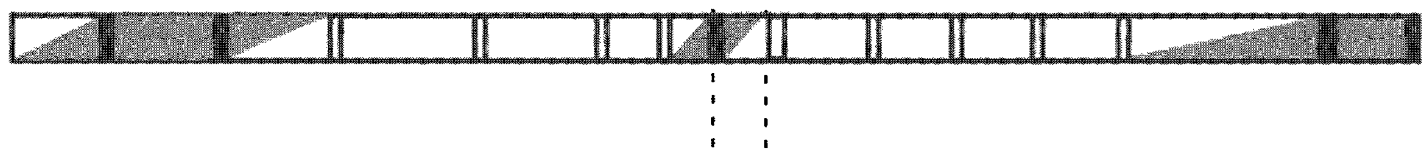

A1(2)

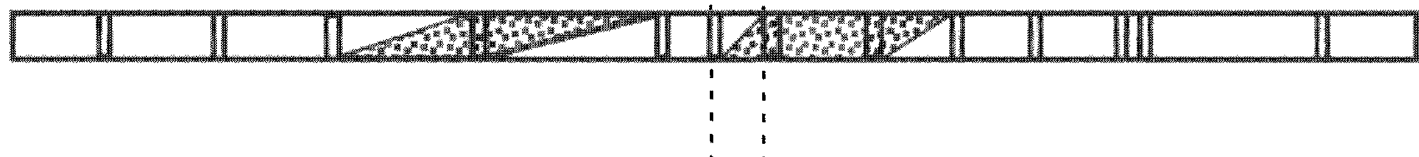

K1362(7)

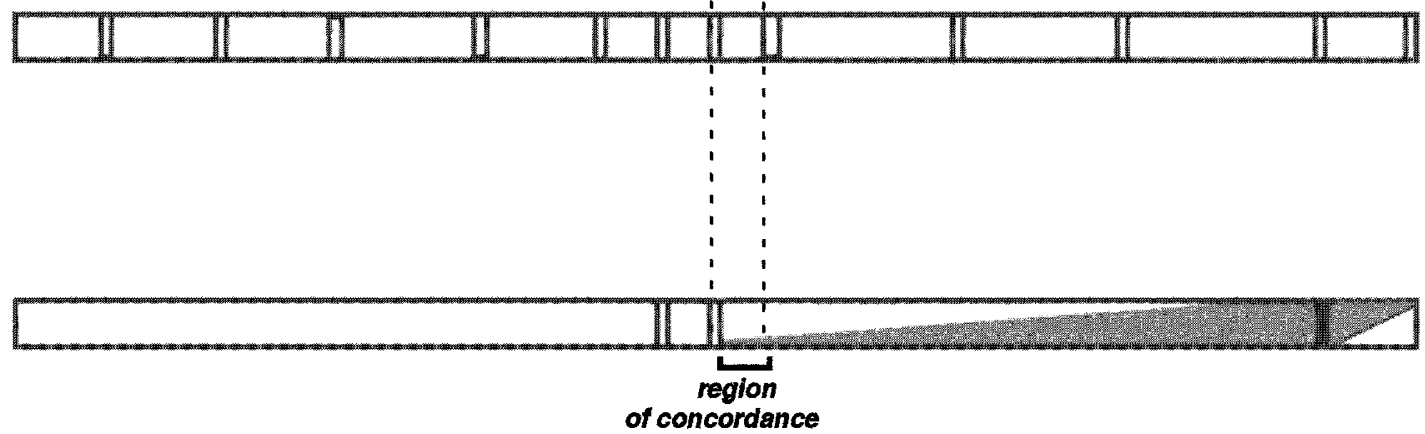

b) $\quad K 1362(7)$

of concordance

\section{1. concordance for grandmaternal allele}

NW concordance for grandpaternal allele

1.7.

Figure 3 Summarized haplotypes of skewed siblings. Family codes and number of informative siblings included in the analysis are displayed on the left, with number of informative siblings in parentheses. E ach rectangle corresponds to a region of the $X$ chromosome in which one or more loci were scored. A t least one marker of those mapping to the corresponding region (Figure 1) was typed in each family. No rectangle appears when the family was not informative or no D NA was available for additional analysis. No information on the grandparental origin of the alleles was available for family $A 1$. a) represents the maternal $X$ chromosome (there is no locus at which all seven daughters in K 1362 are concordant). b) shows the extent of the region for which all seven granddaughters in K 1362 may be concordant for the $X$ chromosome that was preferentially active in their paternal grandmother. The grandmother and granddaughters are concordant at FRAXA and are discordant at A R and XIST ( see also N aumova et al ${ }^{17}$ ). 
A)

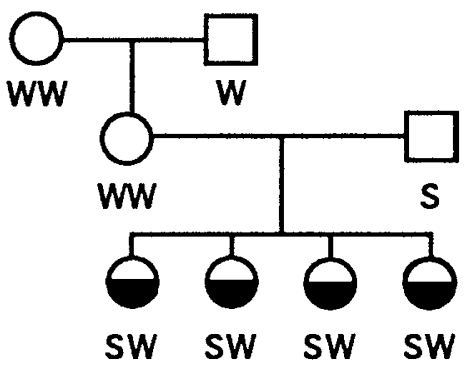

B)
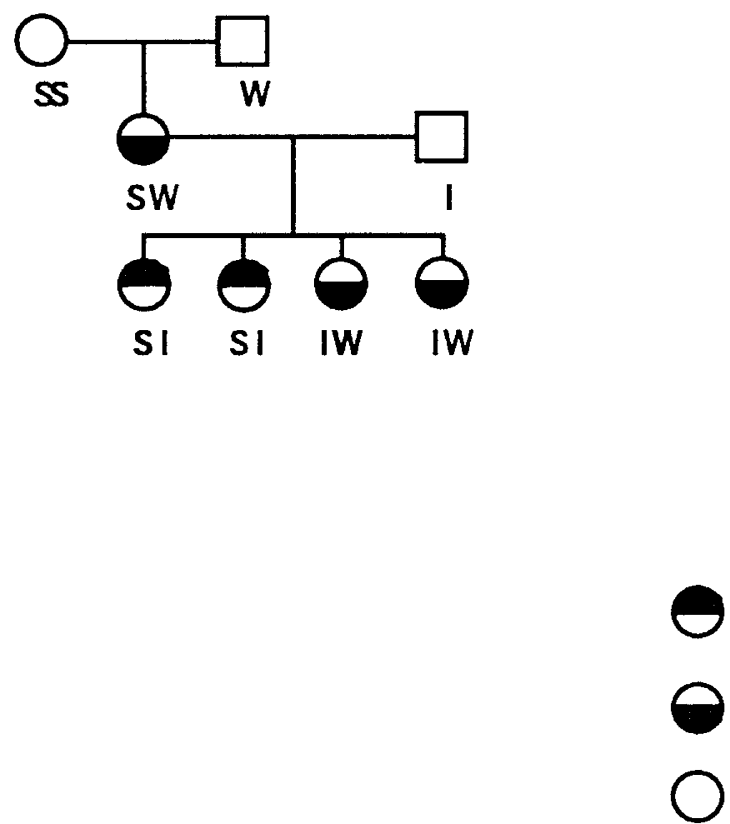

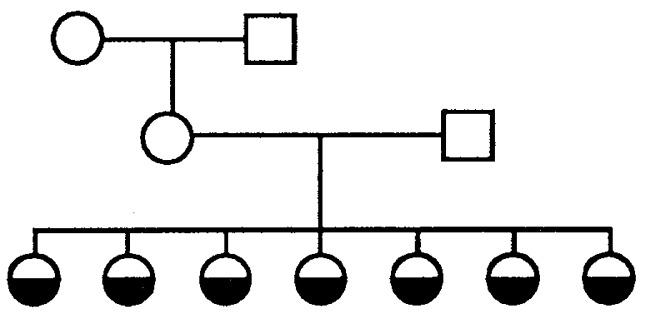

K1362

$S$ (strong) patemal allele active

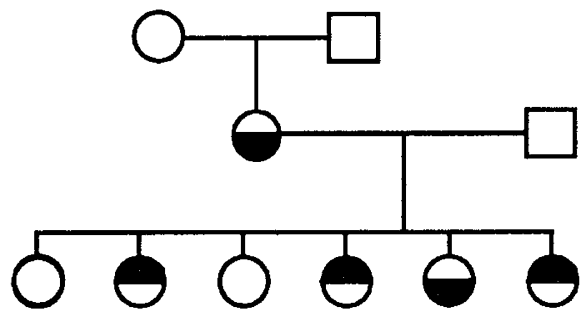

K1341

Figure 4 Skewed $X$ chromosome inactivation in human females as a result of interaction of $X$ ce-like alleles of different strength. A) $M$ odel pedigree (left) with a mother homozygous for a weak allele and a father bearing a strong allele. All the daughters will have a skewed X-inactivation phenotype with their paternal X chromosome active. K 1362 may be an example of such a family. B) M odel pedigree with three $X$ ce-like alleles of different strength. A Il the daughters are predicted to have a skewed $X$-inactivation phenotype, but some of the daughters will have the maternal $X$ chromosome active, and some daughters will have the paternal $X$ chromosome active. K 1341 may be an example of such a family. 
not excluded by this analysis is defined on the proximal side by the marker DX S441 (at Xq13) and on the distal side (at X q21) by the marker DXS3. We note that DXS441 is slightly distal to the XIST locus ${ }^{40}$ but is within the cytogenetic region defined as the XIC. D X S441 is also one of the loci that exhibited linkage to the quantitative trait in the $\mathrm{H}$ aseman-E Iston sib-pair analysis (Table 3).

In terms of postulating a mode of inheritance for the discrete trait, we note that of the $\mathrm{X}$ chromosome-based genetic models that we considered, only one, the mouse $X$ ce model, ${ }^{8,41}$ appears capable of reconciling all of the observations. If alleles at an Xce-like locus in the human behave in the same way as those found in the mouse, then only individuals who are heterozygous for alleles of different 'strength' are predicted to exhibit non-random $X$-inactivation. $X$ chromosomes bearing 'strong' $X$ ce alleles have a high probability of remaining active, whilst $X$ chromosomes bearing 'weak' X ce alleles have a low probability of remaining active. ${ }^{41} \mathrm{This}$ mode of inheritance is predicted to give rise to several unusual familial aggregations of $\mathrm{X}$-inactivation phenotypes. For example, if a mother is homozygous for a weak $X$ ce allele, she will exhibit random $X$-inactivation, but if the father of her daughters carries a strong $X$ ce allele, all of their daughters will be skewed because they will be heterozygous for alleles of different strength. In such a family, of which K 1362 may serve as an example (Figure 4), the daughters are predicted to exhibit the skewed phenotype regardless of which $X$ chromosome they inherit from their mother. No mother-daughter correlation is expected because the mother will have random $\mathrm{X}$-inactivation, whilst all of her daughters are predicted to be skewed. However, sisters in families such as K 1362 are expected to show correlation in both the DS score (because they will all be skewed) and the $\mathrm{PA}_{\text {mat }}$ score (because daughters will tend to have an active paternal chromosome, regardless of which maternal $X$ they have inherited.

Perhaps the most unusual type of family one might expect under this model is that in which some siblings are skewed in favor of an active maternal $X$ whilst others are skewed in favor of an active paternal $X$. This circumstance is predicted to occur as a result of mating between a skewed mother, who was heterozygous for a strong allele and a weak allele, and a father who carried an allele of intermediate strength. Their daughters could be either of two genotypes: heterozygous for a strong allele and an intermediate allele, or heterozygous for a weak allele and an intermediate allele.
D aughters of the first genotype would be skewed in favor of an active maternal $X$, whilst daughters of the second genotype would be skewed in favor of an active paternal X. Data gathered on the four skewed daughters of K 1341 are consistent with such an inheritance pattern in the vicinity of the XIC (Figures 3 and 4 and data not shown).

Our genetic analysis of the $\mathrm{X}$-inactivation phenotypes of females from 38 normal families disproves the hypothesis that $X$-inactivation in human females is 'random'. A Ithough the precise mode by which this trait is inherited is uncertain, our data support a model in which $\mathrm{X}$-inactivation phenotype is expressed as the result of the inheritance of specific alleles at one or two loci on the X-chromosome. In addition, our results are consistent with the existence of a human homologue of the mouse $X$ ce locus.

\section{Acknowledgements}

This work was supported by grants from the A merican Cancer Society (to CS), the National Institutes of $\mathrm{H}$ ealth (GM 52332, to CS) and the Canadian Genetic Diseases Network (NCE program, to KM). A KN and LO contributed equally to this work.

\section{References}

1 Lyon M F : Gene action in the $X$ chromosome of the mouse (M us musculus L.). Nature 1961; 190: 372-373.

2 Belmont JW: Genetic control of $X$ inactivation and processes leading to $\mathrm{X}$-inactivation skewing. $\mathrm{Am} \mathrm{J} \mathrm{H}$ um G enet 1996; 58: 1101-1108.

3 Ropers H-H, Wolff G, Hitzeroth HW: Preferential X inactivation in human placenta membranes: Is the paternal $X$ inactive in early embryonic development of female mammals. H um G enet 1978; 43: 265-273.

$4 \mathrm{H}$ arrison K B, Warburton D: Preferential $X$ chromosome activity in human female placental tissues. Cytogenet $C$ ell G enet 1986; 41: 163-168.

$5 \mathrm{H}$ arrison $\mathrm{KB}: \mathrm{X}$ chromosome inactivation in the human cytotrophoblast. Cytogenet Cell G enet 1989; 52: 37-41.

6 West JD, Frels WI, Chapman VM, Papaionnou VE: Preferential expression of the maternally derived $X$ chromosome in the mouse yolk sac. Cell 1977; 12: 873-882.

7 Takagi N, Sasaki M: Preferential inactivation of the paternally derived $X$ chromosome in the extraembryonic membranes of the mouse. Nature 1975; 256: 640-641.

8 Cattanach BM, Perez JN, Pollard CE: Controlling elements in the mouse genome. Genet Res 1970; 15: 189-195.

9 Willard HF: The sex chromosomes and X-chromosome inactivation. In: The metabolic and molecular bases of inherited disease. 7th edition, Scriver $C R, B$ eaudet $A L$, Sly WS, Valle D. Eds. M cG raw-Hill, Inc., 1995, 719-737. 
10 Plenge R M , Hendrich BD, Schwartz $C$ et al: A promoter mutation in the XIST gene in two unrelated families with skewed X-chromosome inactivation. Nat G enetics 1997; 17: 353-356.

11 Brown $C J$, Ballabio $A, R$ upert $J L$ et al: $A$ gene from the region of the human $X$-inactivation center is expressed exclusively from the inactive $X$ chromosome. Nature 1991; 349: $38-44$

12 Clarke JTR, Greer WL, Strasberg PM, Pearce RD, Skomorowski MA, R ay PN: H unter disease (mucopolysaccaridosis type II) associated with unbalanced inactivation of the $X$ chromosomes in a karyotypically normal girl. A m J Hum G enet 1991; 49: 289-297.

13 Clarke JTR, Wilson PJ, M orris CP et al: Characterization of a deletion at Xq27-q28 associated with unbalanced inactivation of the nonmutant $X$ chromosome. A m J H um G enet 1992; 51: 316-322.

14 Schmidt M, Certoma A, Du Sart D et al: U nusual X chromosome inactivation in a mentally retarded girl with an interstitial deletion $X$ q27: implications for the fragile $X$ syndrome. $\mathrm{H}$ um G enet 1990; 84: 347-352.

$15 \mathrm{D}$ ahl $\mathrm{N}, \mathrm{Hu} \mathrm{L}$ J, Chery $\mathrm{M}$ et al: M yotubular myopathy in a girl with a deletion at X q27-q28 and unbalanced $\mathrm{X}$-inactivation assigns the M TM 1 gene to a $600-\mathrm{kb}$ region. A m J Hum G enet 1995; 56: 1108- 1115.

16 Pegoraro E, Whitaker J, Mowery-R ushton P, Surti V, $L$ anasa $M, H$ offman $E P$ : Familial skewed $X$ inactivation: a molecular trait associated with high spontaneous-abortion rate maps to X q28. Am J H um G enet 1997; 61: 160-170.

17 Naumova A K, Plenge RM, B ird L M et al: Heritability of $X$-chromosome inactivation phenotype in a large family. A m J Hum G enet 1996; 58: 1111-1119.

18 Puck JM, Stewart CC, Nussbaum RL: Maximum-likelihood analysis of human $T$-cell $X$-chromosome inactivation patterns: normal women versus carriers of $X$-linked severe combined immunodeficiency. A m J Hum Genet 1992; 50: 742-748.

$19 \mathrm{M}$ igeon $\mathrm{B} R$ : The postulated $\mathrm{X}$-inactivation center at $\mathrm{X}$ q27 is most reasonably explained by ascertainment bias: heterozygous expression of recessive mutations is a powerful means of detecting unbalanced $X$ inactivation. A m J Hum G enet 1993; 52: 431-432.

20 Nance WE: Genetic tests with a sex-linked marker: glucose-6-phosphate dehydrogenase. Cold Spring $\mathrm{H}$ arbor Symp Q uant Biol 1964; 29: 415-424.

21 Pegoraro E, Schimke R N, A rahata $K$ et al: Detection of new paternal gene mutations in isolated cases of dystrophinopathy in females. Am J Hum Genet 1994; 54: 989-1003.

$22 \varnothing$ rstavik KH, Ø rstavik RE, Eiklid K, Tranebjærg L: Inheritance of skewed $X$ chromosome inactivation in a large family with an $X$-linked recessive deafness syndrome. A m J M ed G enet 1996; 64: 31-34.

23 Allen RC, Zoghbi HY, Moseley AB, Rosenblatt HM, Belmont JW: Methylation of $\mathrm{H}$ pall and $\mathrm{H}$ hal sites near the polymorphic CAG repeat in the human androgenreceptor gene correlates with $X$-chromosome inactivation. A m J Hum G enet 1992; 51: 1229-1239.
24 Naumova A K, O lien L, Bird L M et al: Transmission-ratio distortion of $X$ chromosomes among the male offspring of females with skewed X-inactivation. D ev G enet 1995; 17: 198-205.

$25 \mathrm{Fu} \mathrm{YH}$, Kuhl DPA, Pizzuti A et al: Variation of the CGG repeat at the Fragile $X$ site results in genetic instability: resolution of the Sherman paradox. Cell 1991; 67: 1047-1058.

26 Donner A, Eliasziw M: M ethodology for inferences concerning familial correlations: a review. J Clin E pidemiol 1991; 44: 449-455.

27 Efron B, Tibshirani R: An Introduction to the B ootstrap. M onographs on Statistics and A pplied Probability N o. 57. Chapman \& Hall: N ew York, 1993.

28 R osner $B$, D onner A, Hennekens $\mathrm{CH}$ : Estimation of interclass correlation from familial data. J Roy Stat Soc, Series C (A pplied Statistics) 1977; 26: 179-187.

$29 \mathrm{H}$ aseman JK, Elston RC: The investigation of linkage between a quantitative trait and a marker locus. Behav G enet 1972; 2: 3-19.

30 H auser E R, B oehnke M, G uo SW, R isch N : A ffected-sibpair interval mapping and exclusion for complex genetic traits: sampling considerations. G enet E pidemiol 1996; 13: 117-137.

31 Fain PR, Kort EN, Chance PF et al: A 2D crossover-based map of the human $X$ chromosome as a model for map integration. N at G enet 1995; 9: 261-266.

$32 \mathrm{Ott} \mathrm{J}$. A nalysis of $\mathrm{H}$ uman $\mathrm{G}$ enetic L inkage. John $\mathrm{H}$ opkins U niversity Press, B altimore, 1991

33 G uo SW, Thompson EA : A nalysis of sparse contingency tables: M onte Carlo estimation of exact P-values, U niversity of Washington, D epartment of Statistics, Technical Report NO. 187.

34 Busque L, Mio R, Mattioli J et al: Nonrandom $X$-inactivation patterns in normal females: Iyonization ratios vary with age. Blood 1996; 88: 59-65.

35 Lander $E$, Kruglyak L: G enetic dissection of complex traits: guidelines for interpreting and reporting linkage results. N at G enet 1995; 11: 241-247.

36 Witte J, Elston R, Schork N: Genetic dissection of complex traits. N at G enet 1996; 12: 355-357.

37 Lander $E$, Kruglyak L: G enetic dissection of complex traits, R eply. Nat G enet 1996; 12: 357-358.

38 R ussel LB: Mammalian X-chromosome action: inactivation limited in spread and in region of origin. Science 1963; 140: 976-978.

39 M attei M G, Mattei JF, Vidal I, G irauld F: Structural anomalies of the $X$ chromosome and inactivation center. H um G enet 1981; 56: 401-408.

40 Cuttichia A J, Pearson PL (eds): Human Gene Mapping 1993 J ohn H opkins U niversity Press: Baltimore, 1994.

41 Cattanach BM, Beechey CV: A utosomal and X chromosome imprinting. D evelopment (suppl) 1990; 63-72.

42 Vetrie $D$, Bobrow $M, H$ arris $A$ : Construction of a 5.2-megabase physical map of the human $X$ chromosome at $\mathrm{Xq22}$ using pulsed-field gel electrophoresis and yeast artificial chromosomes. G enomics 1993; 15: 631-642. 